\title{
REANASTOMOSIS TUBARIA LAPAROSCÓPICA POST ESTERILIZACIÓN: TÉCNICA DE TRES PUNTOS
}

\author{
Guillermo Durruty V. ${ }^{1}$, Aída Pinto A. ${ }^{1}$, Ingrid Cárdenas R. ${ }^{1}$, Rodrigo Macaya P. ${ }^{1}$, \\ Alejandro Manzur Y. ${ }^{1}$
}

1 Unidad de Reproducción Humana, Departamento de Obstetricia y Ginecología, Facultad de Medicina, Pontificia Universidad Católica de Chile.

\section{RESUMEN}

Antecedentes: Los avances en las técnicas de cirugía laparoscopia han permitido a cirujanos entrenados realizar reanastomosis tubaria bilateral por laparoscopia, reportando resultados similares a los de la técnica vía laparotómica. Objetivo: Evaluar una nueva técnica de reanastomosis tubaria llamada "técnica de tres puntos". Método: Se realiza estudio retrospectivo que evalúa 5 casos de pacientes con antecedentes de esterilización quirúrgica, a quienes se les realizó reanastomosis tubaria laparoscópica con la técnica de tres puntos. Resultados: La edad promedio de las pacientes fue de 35,8 \pm 5,7 años (rango: $26-40$ años); $80 \%$ $(8 / 10)$ de las trompas fueron reanastomosadas. En dos pacientes la reanastomosis fue unilateral por segmento distal ausente de la trompa contralateral. El tiempo promedio de duración de la cirugía fue de $150 \pm$ 7,9 minutos (rango: 140-160 minutos). La tasa de embarazo fue de $40 \%$ (2/5). Los embarazos se produjeron en las pacientes más jóvenes y con reanastomosis bilateral. En aquellas que no lograron embarazo se realizó histerosalpingografía verificándose el 100\% de las trompas reanastomosadas permeables.

\section{PALABRAS CLAVE: Reanastomosis tubaria, infertilidad, esterilización, ligadura tubaria, laparoscopia, repermeabilización}

\section{SUMMARY}

Background: Advances in endoscopic surgical techniques have allowed well-trained and skilled surgeons to perform tubal anastomosis by laparoscopy reporting similar results than by laparotomy. Objective: To evaluate a new technique we called "three-stitch method". Method: The outcomes for this evaluation were tubal patency and pregnancy rate. A retrospective analysis of five cases of previous tubal sterilization underwent endoscopic tubal reanastomosis using the "three-stitch technique" was done. In all patients a preoperative hysterosalpingogram was performed to confirm occlusion. Results: The average age was $35.8 \pm 5.7$ years (rango: 26-40 years). All the patients underwent previous tubal sterilization (four cases using Pomeroy technique and one case with Yoon Ring). Eighty percent (8/10) of tubes were reanastomosed. In two cases, due to inadequate conditions of distal or proximal ends of one tube, surgery was performed on the other only. The average surgical time was $150 \pm 7.9$ minutes (range 140-160 minutes). The pregnancy rate was $40 \%(2 / 5)$. Pregnancy was achieved in younger patients who underwent bilateral reanastomosis. In those patients where pregnancy was not accomplished, a hysterosalpingography was taken to evaluate tubal patency. All of the reanastomosed tubes showed tubal patency at six months follow-up.

KEY WORDS: Tubal anastomosis, reversal, infertility, sterilization, tubal ligation, laparoscopy 


\section{INTRODUCCIÓN}

Los avances en técnicas quirúrgicas endoscópicas han permitido a cirujanos capacitados y bien entrenados, realizar anastomosis tubaria vía laparoscópica, reportando resultados similares que por vía laparotómica (1).

La técnica clásica de microcirugía por laparotomía consiste en utilizar microscopio o lentes de lupa para realizar la anastomosis. Esta técnica que sigue los principios de la microcirugía, tiene los siguientes pasos: a) colocación de un punto de sutura en mesosálpinx, b) cuatro puntos de sutura de la capa muscular y c) tres o cuatro puntos de sutura en la capa serosa $(2,3)$. Esta técnica realizada por vía laparoscópica presenta algunas dificultades en relación a la manipulación de los instrumentos, magnificación de los tejidos y sobre todo en la realización de las suturas.

Por lo tanto, la búsqueda de técnicas más simples de reanastomosis tubaria ha sido un ítem importante en la cirugía laparoscópica. En este contexto, se ha buscado colocar el mínimo de puntos evitando el punto en el borde inferior de la trompa (4).

El objetivo de este estudio fue evaluar la técnica "de tres puntos", que consiste en realizar un punto en el mesosálpinx y luego tres puntos de sutura (incluyendo capa serosa y muscular) en monocapa en hora 12,4 y 8 . Los resultados evaluados fueron la permeabilidad tubaria y tasa de embarazos.

\section{MATERIAL Y MÉTODO}

Se reclutaron 5 pacientes con esterilización quirúrgica previa con deseo de embarazo, a las que se les realizó reanastomosis tubaria laparoscópica utilizando la técnica de 3 puntos. A cada paciente se le extrajo una muestra de sangre para evaluar niveles de FSH, estradiol, prolactina y TSH, así como también exámenes preoperatorios. A las mayores de 40 años se les solicitó además un electrocardiograma. Todas las pacientes se realizaron una histerosalpingografía para evaluar la cavidad uterina e identificar el lugar de la obstrucción tubaria. A los cónyuges se les solicitó un espermiograma para descartar la presencia de factor masculino.

Técnica quirúrgica. Bajo anestesia general y con la paciente en posición de litotomía, se realiza vaciamiento vesical con sonda Foley y se instala manipulador uterino de Zumi ${ }^{\circledR}$ (Cooper Surgical Inc), que permite movilización uterina e inyección de índigo carmín. Luego de obtener un neumoperitoneo adecuado con $\mathrm{CO} 2$, se coloca trocar umbilical $(10 \mathrm{~mm})$ y se introduce la óptica del laparoscopio conectado a video cámara. Se realizan tres punciones accesorias de $5 \mathrm{~mm}$, una suprapúbica y dos laterales. Se procede a la exploración de la cavidad abdominal y pelvis, realizando adherensiolisis en caso necesario. Se evalúan las trompas de Falopio y se identifica el lugar de la obstrucción. En la primera inspección se evalúan los remanentes tubarios y se verifica la presencia de cabos dístales que puedan encontrarse ausentes en caso de resección tubaria amplia durante la esterilización.

Para la realización del procedimiento se utilizaron instrumentos microquirúrgicos de Koh $₫$ (Karl Storz Company), además de un disector de $5 \mathrm{~mm}$.

La técnica de los tres puntos sigue los principios básicos de la microcirugía que son: magnificación, disección atraumática, hemostasia cuidadosa con bipolar fino $(1 \mathrm{~mm})$ y aproximación precisa de los tejidos, con irrigación continua de los mismos $(5,6)$.

Los pasos a seguir son: disección de mesosálpinx, sección tubaria perpendicular al eje en el punto proximal de la obstrucción. Verificación de la permeabilidad del segmento mediante la inyección de índigo carmín a través de la cánula intrauterina. El segmento tubario distal es seccionado perpendicularmente a su eje en su extremo proximal, se verifica permeabilidad inyectando índigo carmín a través de un catéter de peridural insertado desde proximal a distal. No se realiza canulación proximal así como tampoco se usa el catéter como guía para suturas. Se coloca el primer punto de sutura en mesosálpinx, utilizando Vicryl 5-0 y realizando nudos intracorpóreos, con la intención de alinear apropiadamente ambos segmentos tubarios. Luego se realizan tres puntos de sutura en la trompa siguiendo una distribuacion horaria (en hora 12, 4 y 8), usando Vicryl 6-0 en un solo plano, incluyendo capa serosa y muscular. Se comienza la sutura desde la capa serosa atravesando la muscular, evitando entrar al lumen y se ajusta finalmente con nudos intracorpóreos. Al completar la anastomosis tubaria, se verifica permeabilidad de la misma observando la salida de índigo carmín por la fimbria (Figura $1 \mathrm{~A}$ a $1 \mathrm{H}$ ). Durante todo el procedimiento se irrigan los segmentos comprometidos con solución fisiológica y se realiza hemostasia con pinza bipolar fina.

\section{RESULTADOS}

La edad promedio fue de 35,8 $\pm 5,7$ años (rango: 26-40 años). Cuatro pacientes habían sido esterilizadas por técnica de Pomeroy y una por anillo de Yoon. De las 10 trompas a reanastomosar, solo se logro el procedimiento en 8 (80\%). En dos trompas no fue posible, por presentar segmento distal 


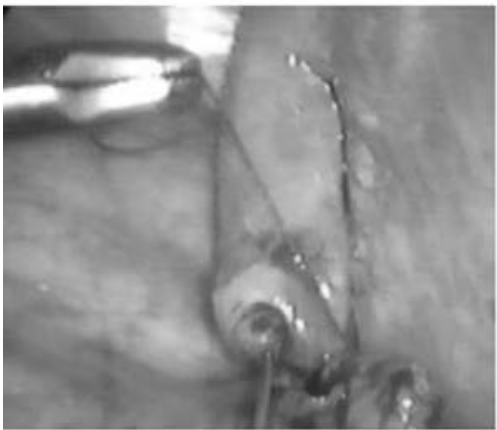

A

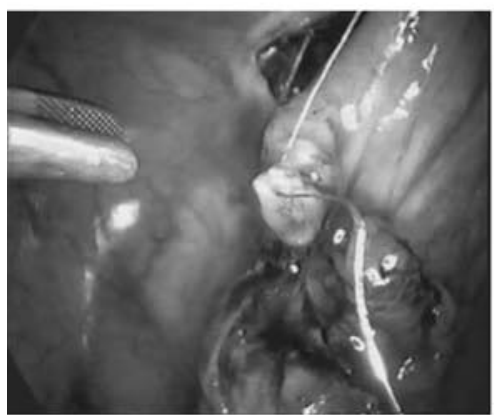

C

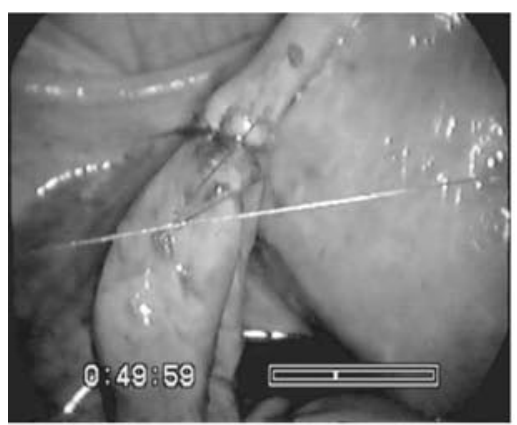

$\mathrm{E}$

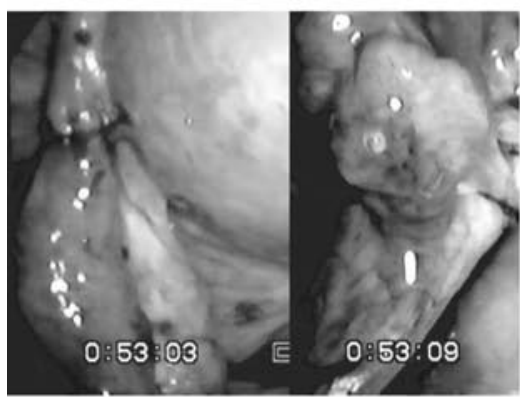

G

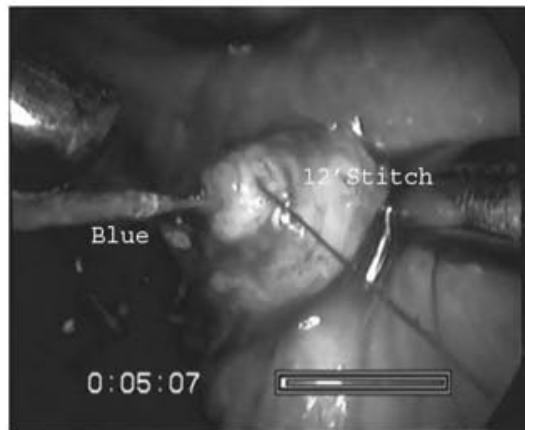

B

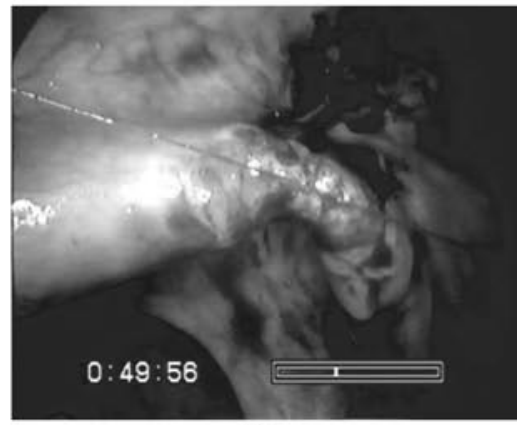

D

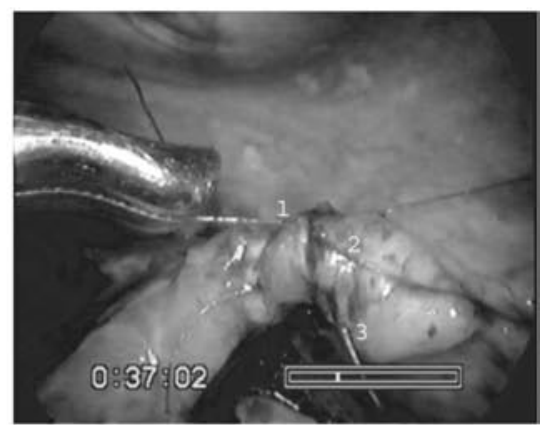

$\mathrm{F}$

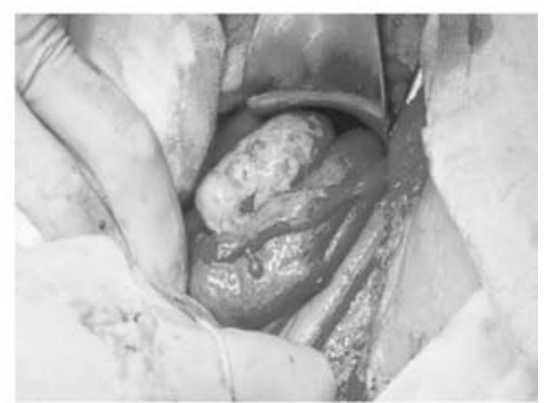

$\mathrm{H}$

Figura 1. A) punto en mesosalpinx; B) verificación de permeabilidad segmento proximal con índigo carmín; C y D) punto en plano seromuscular en hora 12; E) aproximación de ambos segmentos con punto en plano seromuscular en hora 12; F) visualización de los tres puntos en plano seromuscular; G) verificación de permeabilidad tubaria con índigo carmín; H) visualización de reanastomosis tubaria durante cesárea. 
inadecuado (ausencia de fimbria), realizándose en esos casos una anastomosis unilateral. El tiempo quirúrgico promedio fue de $150 \pm 7,9$ minutos (rango: 140-160 minutos). No hubo complicaciones intra ni posoperatorias. El alta fue a las 18-24 horas en todos los casos.

Se embarazaron 2 de 5 pacientes (40\%), ambos embarazos fueron intrauterinos. El embarazo lo lograron las pacientes más jóvenes y en quienes también se había obtenido la reanastomosis bilateral (Tabla I). Los embarazos se lograron a los 2 y 3 meses poscirugía. En aquellas pacientes que no lograron embarazo a los 6 meses del procedimiento se les solicito una histerosalpingografía para evaluar la permeabilidad tubaria, confirmándose que el $100 \%$ de las trompas reanastomosadas estaban permeables a los 6 meses poscirugía (Tabla I).

\section{Tabla I}

\section{RESULTADOS PRIMARIOS: PERMEABILIDAD TUBARIA Y EMBARAZOS}

\begin{tabular}{ccccc}
\hline Casos & Edad & Reanastomosis & Embarazo & $\begin{array}{c}\text { HSG post } \\
\text { operatoria }\end{array}$ \\
\hline A & 26 & bilateral & positivo & no \\
B & 36 & bilateral & positivo & no \\
C & 37 & bilateral & negativo & $\begin{array}{c}\text { permeable } \\
\text { bilateral }\end{array}$ \\
D & 40 & unilateral & negativo & permeable \\
E & 40 & unilateral & negativo & permeable
\end{tabular}

HSG: histerosalpingografía.

\section{DISCUSIÓN}

Los cambios socioculturales y médicos han permitido que mujeres de mayor edad deseen lograr un embarazo, y entre ellas, muchas mujeres esterilizadas a quienes se les ofrece la reanastomosis tubaria microquirúrgica (6).

Las causas del arrepentimiento por la esterilización quirúrgica, son haber realizado el procedimiento a mujeres jóvenes, cambio en el estado civil (nueva pareja), muerte de algún hijo, bajo nivel socioeconómico y/o cultural, entre otros (7).

Desde 1913 se han llevado a cabo procedimientos de anastomosis tubaria laparotómica, siendo inicialmente la tasa de embarazo muy baja. En 1960 los avances en medicina desarrollaron técnicas microquirúrgicas que incrementan las tasas de embarazo, llegando a 50-75 \%, como lo demuestran varios reportes $(3,8-11)$.

La evolución de la cirugía endoscópica y el de- sarrollo del instrumental endoscópico, han permitido la realización de estas técnica vía laparoscópica con resultados variables y progresivamente mejores $(13,14)$.

En 1989, Sedbon y cols (12), reportaron el primer embarazo intrauterino. El procedimiento consistió en utilizar una guía intraluminal y un pegamento biológico en una sola trompa vía laparoscópica, a pesar de que esta técnica era mas atractiva, por el menor tiempo de hospitalización, menor dolor y mejor recuperación postoperatoria, los resultados en relación a fertilidad fueron menos exitosos que los de la técnica micro quirúrgica vía laparotómica. Así lo demostró Reich y cols (13), quienes en 1993 reportaron 22 casos de reanastomosis laparoscópica, utilizando una técnica de 2 puntos e instrumental laparoscópico convencional de $5 \mathrm{~mm}$, con una tasa de embarazo intrauterino de $35 \%$, tasa de embarazo aún baja comparado a lo reportado por laparotomía (50-70\%).

Con la aparición de instrumental microquirúrgico de Koh las tasas de embarazo mejoraron. En 1995, Koh y Janik (14), publicaron la primera anastomosis tubaria con técnica microquirúrgica laparoscópica, utilizando instrumental de $3 \mathrm{~mm}$, especialmente diseñado para el procedimiento. La tasa de embarazo obtenida fue de $71 \%$. La técnica utilizada fue la de 4 puntos colocados en horas 12, 3, 6, 9 suturando la capa muscular con prolene o nylon 60 , previo punto de aproximación en mesosalpinx y terminar con puntos separados la serosa.

Yoon y cols (15), en 1999, reportan una tasa de embarazo de $84,9 \%$ con la técnica de anastomosis en dos capas con 4 puntos de sutura en cada una y con sutura intermitente en la serosa, utilizando material de monofilamento 7-0 u 8-0 para la sutura de la capa muscular. En los primeros casos realizados con esta técnica el tiempo operatorio fue de 4 a 5 horas, mejorando con la práctica y llegando a un promedio de 120 minutos.

Publicaciones posteriores con la misma técnica reportan tasas de embarazo que varían entre 65$80 \%$ aproximadamente $(1,16)$. Una vez solucionado el problema del instrumental quirúrgico inadecuado y contando con una mejor visión, una nueva meta fue la reducción del tiempo quirúrgico.

Dada la dificultad en la realización de las suturas vía laparoscópica es que se ha intentado modificar la técnica quirúrgica original. Dubuisson en 1998 (17), siguiendo el concepto de la técnica microcirugía, publica la técnica de "un punto", que consiste en que luego de la preparación y aproximación de los 2 segmentos tubarios con un punto en el mesosalpinx, se realiza la anastomosis con un solo punto de sutura en hora 12 del borde an- 
timesenterico de la trompa incluyendo en la sutura la capa muscular y serosa, utilizando vicryl 6-0 o 7-0, reportando una tasa de embarazo de $53,1 \%$ y tiempo quirúrgico promedio de 202,5 minutos (rango: 180-240 minutos).

En nuestra breve experiencia, iniciamos la técnica laparoscópica en el 2000 , realizando los primeros casos con la colocación de 4 puntos seromusculares y utilizando instrumental laparoscópico tradicional de $5 \mathrm{~mm}$. A raíz de lo difícil de la manipulación tubaria con el instrumental clásico, y de la frecuente rotura del vicryl 6.0 con los portagujas tradicionales, las cirugías eran de muy larga duración (mayor a 3 horas). Entonces realizamos un caso con la técnica de 1 punto descrita por Dubuisson y la impresión intraoperatoria fue satisfactoria, sin embargo, la ausencia de embarazo y la visualización de una trompa obstruida en la histerosalpingografía de control, nos hizo cuestionar esta técnica.

Considerando las bajas tasas de embarazo obtenidas en el trabajo de Dubuisson y la no modificación de tiempos operatorios, planteamos simplificar la técnica original, colocando solo tres puntos seromusculares, evitando así la realización del punto por la cara inferior de la trompa, el cual es el más difícil de realizar por vía laparoscópica, y por ende el mas lento. Los resultados obtenidos con esta técnica en relación a la tasa de embarazo y permeabilidad tubaria son concordantes con los publicados y con tiempos operatorios aceptables (17). En nuestro conocimiento, no existen trabajos con esta misma técnica.

Diferentes autores han reportado algunos factores pronósticos en el éxito de la cirugía; entre ellos se describe el método de esterilización utilizado, tiempo transcurrido entre la esterilización y el momento de reanastomosis, lugar de la reanastomosis, edad, longitud de la trompa y número de trompas reanastomosadas; siendo la edad y la longitud de la trompa los que presentaron diferencias estadísticamente significativas $(18,19,20)$.

En relación a la edad, en las pacientes menores de 35 años la tasa acumulativa de embarazo intrauterino es $70 \%$, observando el logro de la mayoría de los embarazos dentro de los primeros 18 meses poscirugía. Dubuisson y cols (10), evaluaron las tasas de embarazo según la edad de la paciente, demostrando que la edad es el factor predictor de embarazo más importante; los resultados obtenidos fueron $82,5 \%$ en mujeres menores de 33 años a los dos años de la cirugía, y $51,4 \%$ en mujeres mayores de 40 años. Trimbos-Kempers (21), realizó reanastomosis en pacientes mayores de 40 años, reportando una tasa de embarazo de $45 \%$. Glock y cols (22), reportan tasa de parto en pacientes ma- yores de 40 años de 14,3\%.

Una pregunta importante es si ante infertilidad por esterilización quirúrgica es mejor la fertilización in vitro con embrio transferencia (FIV) o cirugía reconstructiva. Las tasas de embarazo por ciclo de FIV van del 21 al $46 \%$ en pacientes con patología tubaria $(23,24)$. El Programa Europeo de Fertilización in vitro (ESHRE) reporta $27 \%$ de embarazos y los EEUU 36,5\%, sin discriminar las patologías de la paciente $(25,26)$. No hay trabajos comparativos al respecto, pero las publicaciones reportan tasa de embarazos más satisfactorias con la cirugía reconstructiva como también menores complicaciones que con un ciclo de FIV.

En nuestra opinión las pacientes menores de 35 años, cuya única causa de infertilidad es la esterilización quirúrgica previa, debieran tener indicación de reanastomosis tubaria. Por el contrario pacientes con pobre pronóstico reproductivo, evaluado desde su reserva ovárica, endometriosis severa $u$ otro factor asociado, como por ejemplo factor masculino, debieran ir directamente a procedimientos de reproducción asistida.

Se debe enfatizar la necesidad de cirujanos entrenados en cirugía laparoscópica avanzada, para realizar este procedimiento.

\section{CONCLUSIONES}

La reanastomosis tubaria laparoscópica es una alternativa válida a la técnica convencional laparotómica. Los avances en la cirugía laparoscópica han permitido mejorar las técnicas quirúrgicas al igual que la tasa de éxito en términos de embarazos. La edad de la paciente continúa siendo un factor determinante en los resultados. La técnica de los "tres puntos" muestra resultados similares a los publicados en la literatura en relación a la tasa de embarazo y permeabilidad tubaria. Probablemente un mayor número de casos analizados nos permitirá validar esta técnica.

\section{BIBLIOGRAFÍA.}

1. Cha SH, Lee MH, Kim JH, Lee CN, Yoon TK, Cha KY. Fertility outcome after tubal anastomosis by laparoscopy and laparotomy. J Am Assoc Gynecol Laparosc 2001;8(3):348-52.

2. Winston RM. Microsurgical tubocornual anastomosis for reversal of sterilisation. Lancet 1977;1:284-5.

3. Gomel V. Microsurgical reversal of female sterilization: a reappraisal. Fertil Steril 1980;33(6):587-97.

4. Dubuisson JB, Swolin K. Laparoscopic tubal anastomosis (the one stitch technique): preliminary results. Hum Reprod 1995;10(8):2044-6.

5. Swolin K. Contribution to the surgical treatment of fe- 
male sterility: experimental and clinical studies. Acta Obstet Gynecol Scand 1967;46(14):1-20.

6. Gomel V. Tubal reconstruction by microsurgery. Fertil Steril 1977;28(1):59-65.

7. Siegler AM, Hulka J, Peretz A. Reversibility of female sterilization. Fertil Steril 1985; 4(43):499-510.

8. Kim SH, Moon SY, Shin CJ, Lee JY, Kim JG, Chang YS. Microsurgical reversal of tubal sterilization: A report on 1118 cases. Fertil Steril 1997;68: 865-70.

9. Kim JD, Kim KS, Doo JK, Rhyeu CH. A report on 385 cases of microsurgical tubal reversals. Fertil Steril 1997;68:875-80.

10. Dubuisson JB, Chapron C, Nos C, Morice P, Aubriot FX, Garnier P. Sterilization reversal: Fertility results. Hum Reprod 1995;10:1145-51.

11. DeCherney AH, Mezer HC, Naffolin F. Análysis of failure of microsurgical anastomosis after midsegments, no coagulation tubal ligation. Fertil Steril 1993;39:61822.

12. Sedbon E, Bouquet de Joliniére J, Boudouris O, Madelenat $P$. Tubal desterilization through exclusive laparoscopy. Hum Reprod 1989;4:158-9.

13. Reich H, McGlynn F, Parente C, Sekel L, Leive M. Laparoscopic tubal anastomosis. J Am Assoc Gynecol Laparosc 1993;1:16-9.

14. Koh CH, Janik GM. Laparoscopic microsurgical tubal anastomosis. Obstet Gynecol Clin North Am 1999;26(1):189-200.

15. Yoon TK, Sung HR, Kang HG, Cha SH, Lee CN, Cha KL. Laparoscopic tubal anastomosis: Fertility outcome in 202 cases. Fertil Steril 1999;72:1121-6.

16. Hawkins J, Dube D, Kplow M, Tulandi T. Cost analysis of tubal anastomosis by laparoscopy and by laparotomy. J Am Assoc Gynecol Laparosc 2002;9:120-4.

17. Dubuisson JB, Chapron C. Single suture laparosco- pic tubal reanastomosis. Curr Opin Obstet Gynecol 1998;10(4):307-13.

18. Miranda C, Carvajal A, Venditti P. Laparoscopic tubal reanastomosis. Gynecol Surg 2005;2:313-5.

19. Rouzi AA, Mackinnon M, McComb PA. Predictors of success of reversal of sterilization. Fertil Steril 1995;64:29-36.

20. Silber SJ, Cohen RS. Microsurgical reversal of female sterilization: The role of the tubal length. Fertil Steril 1980;33:598-601.

21. Trimbos-Kemper TCM. Reversal of sterilization in women over 40 years of age: A multicenter survey in the Netherlands. Fertil Steril 1990;53:575-7.

22. Glock JL, Hunt RB, Kim AH, Trad FS, Hulka JF, Brumsted JR. Reproductive outcome alter tubal reversal in women 40 years of age or older. Fertil Steril 1996;65:863-5.

23. Holst N, Maltau JM, Forsdahl F, Hansen LJ. Handling of tubal infertility alter introduction of in vitro fertilization: changes and consequences. Fertil Steril 1991;55:140-2.

24. Chardon C, Boulieu D, Rochet Y, Payan F, Ayzac L. Stèrilité d'origine tubaire: quel traitement proponer: FIV ou chirurgie tubaire? Rev Fr Gynécol Obstét 1992;87:355-60.

25. The European IVF-monitoring programme (EIM), for the European Society of Human Reproduction and Embryology (ESHRE). Assisted technology in Europe, 1998. Hum Reprod 2001;16(11):2459-71.

26. Assisted reproductive technology in the United States. 1997 results generated from the American Society for Reproductive Medicine/Society for Assisted Reproductive Technology Registry. Fertil Steril 2000; 74(4):641-53. 